\title{
Untersuchungen zur Ermittlung der Bruchzähigkeit an MDF-Platten
}

\author{
P. Niemz, M. Diener, E. Pöhler
}

Die Bruchzähigkeit ist eine Materialkenngrösse, die das Verhalten eines Werkstoffes gegenüber der Ausbreitung eines Risses kennzeichnet. In dieser Arbeit wurde der Einfluß von Rohdichte und Holzfeuchte auf die Bruchzähigkeit von MDF untersucht. Die Prüfung erfolgte nach ASTM E 399 an CT-Proben (Kompaktzugproben) durch Zugbelastung parallel zur Plattenebene. Zur Beurteilung des Bruchverhaltens und des Bruchbildes wurde zusätzlich die Schallemissionsanalyse und die Rasterelektronenmikroskopie eingesetzt. Die Untersuchungen zeigten, daß die Bruchzähigkeit mit zunehmender Rohdichte ansteigt. Eine Erhöhung der Holzfeuchte bewirkt eine deutliche Reduzierung der Bruchzähigkeit. Die Messungen der Schallemission während der Rißaufweitung brachten keine eindeutigen Ergebnisse im Hinblick auf das Bruchverhalten von MDF. Die rasterelektronenmikroskopischen Untersuchungen machten deutlich, daß die Fasern überwiegend aus dem Faserverbund herausgezogen werden. Reine Holzbrüche, wie sie bei Spanplatte z.T. erfolgen, waren hier nicht nachweisbar.

\section{Determination of the fracture toughness of MDF board}

Fracture toughness is a measure for the crack resistance of a material. This paper presents the results of an analysis on the influence of density and moisture content on fracture toughness of MDF. Fracture toughness was measured using ASTM E 399 with CT-specimens (parallel to the board direction). Acoustic emission analysis and scanning electron microscopy (SEM) were used to assess the kind of fracture and the fracture surface. Higher fracture toughness values were found with increasing density. An increase in moisture content caused a decrease in fracture toughness. Acoustic emission analysis showed no clear results regarding the kind of fracture of MDF. According to SEM fibres were nearly torn out of the MDF board. Fractures of fibres, as they occur in particleboard, were not observed.

\section{1}

\section{Einleitung}

Im Rahmen der Einführung neuer Berechnungsmethoden für die Dimensionierung von Holzkonstruktionen gewinnt die Bruchzähigkeit auch in der Holzforschung zunehmend an Bedeutung.
Gegenstand der Bruchmechanik ist die Entwicklung analytischer Modelle des Bruchvorganges sowie von Kenngrößen und Prüfmethoden zur bruchsicheren Gestaltung von Werkstoffen und Bauteilen. Unter der Bruchzähigkeit versteht man den kritischen Spannungsintensitätsfaktor $K_{I C}$, bei dem Gewaltbruch eintritt. Der Wert von $K_{I C}$ gibt Aufschluß darüber, welchen Widerstand ein Material der Ausbreitung eines Risses entgegensetzt. Voraussetzung für einen gültigen $K_{I C}$-Wert, der eine typische Werkstoffeigenschaft darstellt, ist ein Zustand ebener Dehnung und ein linear-elastisches Verhalten der mit einem scharfen Anriß versehenen Probe. Dies ist weitgehend erfüllt, wenn der Durchmesser der plastischen Zone an der Rißspitze klein ist im Verhältnis zu den Probenabmessungen.

Zur Bruchzähigkeit von Holz wurden in den letzten Jahren umfangreiche Arbeiten durchgeführt (Logemann 1991, Boström 1992, Valentin et al. 1991, Stanzl-Tschegg et al. 1995, Erhart et al. 1996). Zur Bruchzähigkeit von Holzwerkstoffen liegen vergleichsweise wenige Arbeiten vor. Niemz und Schädlich (1992) untersuchten den Einfluß struktureller Parameter auf die Bruchzähigkeit von Spanplatten bei Biegebelastung. Angaben zu MDF fehlen.

Im Rahmen der vorliegenden Arbeit wird der Einfluß der Rohdichte und des Feuchtegehaltes auf die Bruchzähigkeit bei Zugbelastung parallel zur Plattenebene bestimmt. Zur Beurteilung des Bruchverhaltens werden zusätzlich die Schallemissionsanalyse und die Rasterelektronenmikroskopie eingesetzt.

\section{2}

\section{Versuchsmaterial und -methodik}

\section{1}

\section{Versuchsmaterial}

Um den Einfluß der Rohdichte auf die Bruchzähigkeit zu untersuchen, wurden industriell gefertigte MDF-Platten unterschiedlicher Nennrohdichten verwendet (superleicht $500 \mathrm{~kg} / \mathrm{m}^{3}$, leicht $600 \mathrm{~kg} / \mathrm{m}^{3}$, standard $\left.680 \mathrm{~kg} / \mathrm{m}^{3}\right)$. An Platten der Dichtekategorie superleicht wurden zusätzlich Untersuchungen zum Einfluß des Feuchtegehaltes durchgeführt Für die Ermittlung des Rohdichteeinflusses wurden je Variante 20 Prüfkörper, für die Ermittlung des Feuchteeinflusses 10 Prüfkörper der Nennrohdichte $500 \mathrm{~kg} / \mathrm{m}^{3}$ verwendet.

\section{2 \\ Versuchsmethodik}

\subsection{1}

\section{Bestimmung der Bruchzähigkeit}

Eine Probe des Werkstoffes mit definiertem Anriß wird belastet, bis Rißwachstum eintritt. Dabei werden die Kraft 
$P$ in der Lastlinie und die Rißaufweitung an der Stirnseite der Probe gemessen. Aus der Spannung $\sigma$, der Rißlänge a und einem Geometriefaktor $Y=f(a / W)$ errechnet sich gemäß der Gleichung

$K=\sigma \times \sqrt{a} \times f\left(\frac{a}{W}\right)$ oder $K=\frac{P}{B \times \sqrt{W}} \times Y$

$K$ Spannungsintensität $[\mathrm{MPa} \times \sqrt{ } m$ ]

$\sigma \quad$ Spannung [MPa]

a Rißlänge [mm]

$P \quad$ Kraft [N]

$B$ Dicke [mm]

$W$ Probenweite [mm]

$Y$ Geometriefaktor $f(a / W)$

die kritische Spannungsintensität, die Bruchzähigkeit $K_{I C}$. Der genaue Versuchsablauf sowie die Bedingungen sind in der Norm ASTM E 399 festgelegt (ASTM 1994).

Die in dieser Arbeit durchgeführten Messungen richteten sich weitgehend nach dieser Vorschrift. Die verwendeten Proben waren sogenannte 'Kompaktzugproben' (CT-Proben). Die Probendicke $B$ war aus herstellungstechnischen Gründen $18 \mathrm{~mm}$, die restlichen Abmessungen entsprachen den in der ASTM angegebenen Werten. Abbildung 1 zeigt die verwendeten Prüfkörper. Die Belastung erfolgte parallel zur Plattenebene. In die Prüfkörper wurde zunächst von der Stirnseite (Schmalfläche) mittels Kreissäge ein $37 \mathrm{~mm}$ tiefer Einschnitt eingebracht, danach wurde der Riß durch einen Schnitt mit einer Rasierklinge auf $38 \mathrm{~mm}$ erweitert.
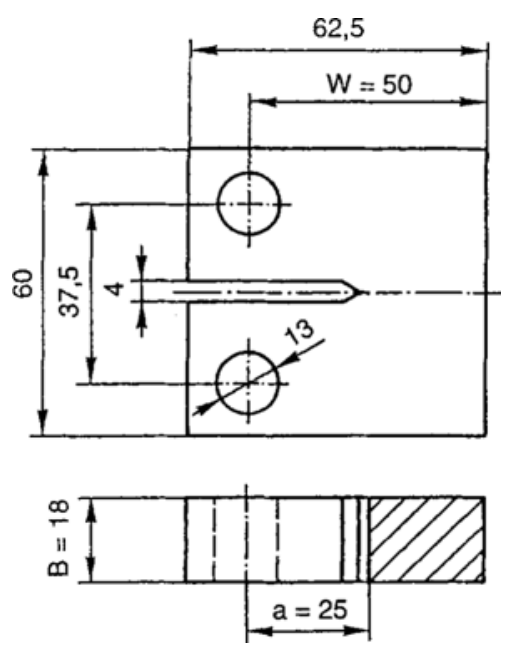

Bild 1. Abmessungen der verwendeten CT-Proben

Fig. 1. Dimensions of the CT-specimens

Tabelle 1. Bruchzähigkeit von MDF unterschiedlicher Rohdichten Table 1. Fracture toughness of MDF with different densities

\begin{tabular}{|c|c|c|c|c|}
\hline Material & $\begin{array}{l}\text { stat. } \\
\text { Kennzahl }\end{array}$ & $\begin{array}{l}\text { Dicke } \\
\text { in } \mathrm{mm}\end{array}$ & $\begin{array}{l}\text { Rohdichte } \\
\text { in } \mathrm{kg} / \mathrm{m}^{3}\end{array}$ & $\begin{array}{l}\text { Bruchzä- } \\
\text { higkeit in } \\
\mathrm{MPa} \mathrm{m}^{1 / 2}\end{array}$ \\
\hline \multirow[t]{2}{*}{ MDF SL } & $x$ & 18 & 489 & 1.2 \\
\hline & $s$ & & 6.1 & 0.09 \\
\hline \multirow[t]{2}{*}{ MDF L } & $\mathrm{x}$ & 18 & 596 & 1.56 \\
\hline & $s$ & & 6.6 & 0.1 \\
\hline \multirow[t]{2}{*}{ MDF STD } & $\mathrm{x}$ & 18 & 684 & 2.15 \\
\hline & $s$ & & 2.7 & 0.19 \\
\hline
\end{tabular}

Die Untersuchungen zum Einfluß der Rohdichte wurden an im Normalklima bei $20^{\circ} \mathrm{C}$ und $65 \%$ relativer Luftfeuchtigkeit klimatisierten Proben durchgeführt.

Zur Ermittlung des Feuchteeinflusses wurden Proben der Nennrohdichte $500 \mathrm{~kg} / \mathrm{m}^{3}$ in einem Klimaschrank bei $20{ }^{\circ} \mathrm{C}$ und einer relativen Luftfeuchtigkeit von $50 \%, 65 \%$, $85 \%$ und $95 \%$ klimatisiert sowie in einem Trockenschrank bei $50{ }^{\circ} \mathrm{C}$ bis auf eine Feuchte von 3,5\% getrocknet.

\subsection{2}

Untersuchungen zur Schallemission bei der Rissaufweitung

Die Schallemissionsmessungen erfolgten nur an den zum Dichteeinfluß durchgeführten Versuchsreihen. Die Schallemissionssignale wurden mit einer Schallemissionsmesskarte SEK 3243 vom Fraunhoferinstitut für zerstörungsfreie Prüfverfahren, Dresden, gemessen.

Für die Vorverstärkung der Signale wurde ein Vorverstärker SEV 3202, als Sensor ein piezoelektrischer Aufnehmer mit einer Resonanzfrequenz von $30-300 \mathrm{kHz}$ verwendet. Der Aufnehmer wurde in Rißnähe mit Maschinenfett an die Holzprobe angekoppelt.

\section{3}

\section{Versuchsergebnisse}

In den Tabellen 1 und 2 sind die Ergebnisse des Rohdichte- und Holzfeuchtigkeits-Einflusses auf die Bruchzähigkeit zusammengefaßt.

Mit zunehmender Dichte der Platten steigt die Bruchzähigkeit erwartungsgemäß an (Bild 2).

Tabelle 2. Einfluß der Feuchtigkeit auf die Bruchzähigkeit von MDF (Nennrohdichte im Normalklima: $500 \mathrm{~kg} / \mathrm{m}^{3}$ )

Table 2. Influence of moisture content on fracture toughness of MDF (density: $500 \mathrm{~kg} / \mathrm{m}^{3}$ )

\begin{tabular}{lll}
\hline $\begin{array}{l}\text { Gleichgewicht- } \\
\text { feuchte in\% }\end{array}$ & $\begin{array}{l}\text { stat. } \\
\text { Kennzahl }\end{array}$ & $\begin{array}{l}\text { Bruchzähigkeit } \\
\text { im MPa m }\end{array}$ \\
\hline 21.4 & $\mathrm{x}$ & 0.36 \\
& $\mathrm{~s}$ & 0.03 \\
14.4 & $\mathrm{x}$ & 0.68 \\
& $\mathrm{~s}$ & 0.06 \\
10.2 & $\mathrm{x}$ & 1.26 \\
& $\mathrm{~s}$ & 0.08 \\
7.7 & $\mathrm{x}$ & 1.33 \\
& $\mathrm{~s}$ & 0.38 \\
3.5 & $\mathrm{x}$ & 1.29 \\
& $\mathrm{~s}$ & 0.06 \\
\hline
\end{tabular}

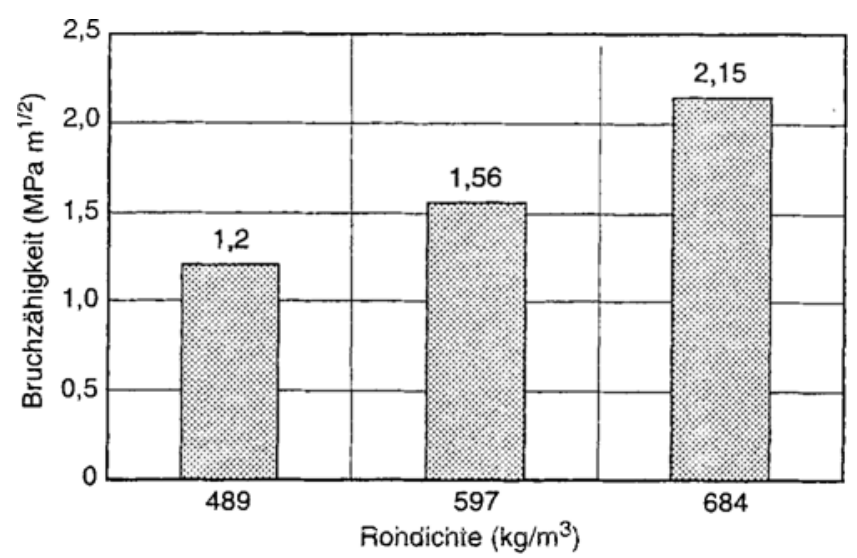

Bild 2. Einfluß der Rohdichte auf die Bruchzähigkeit von MDF Fig. 2. Influence of the density on fracture toughness of MDF 
Eine Erhöhung der Holzfeuchtigkeit bewirkt einen deutlichen Abfall der Bruchzähigkeit. Dieser Abfall ist bei einer Holzfeuchtigkeit oberhalb $10 \%$ stark ausgeprägt. Der Feuchteeinfluß spiegelt sich auch in den Rißaufweitungsdiagrammen wieder (Bild 3a-c). Die Abbildungen zeigen typische Diagramme für in $50 \%, 85 \%$ und $90 \%$ relativer Luftfeuchtigkeit gelagerte Proben. Mit zunehmender Holzfeuchte steigt die Rißaufweitung deutlich an, die Proportionalitätsgrenze sinkt.

Die Schallemission beginnt unmittelbar bei Belastung der Proben und steigt mit zunehmender Rißaufweitung an $(B i l d 4 a-c)$. Eine eindeutige Differenzierung konnte zwischen den einzelnen Dichtestufen allerdings nicht festge-

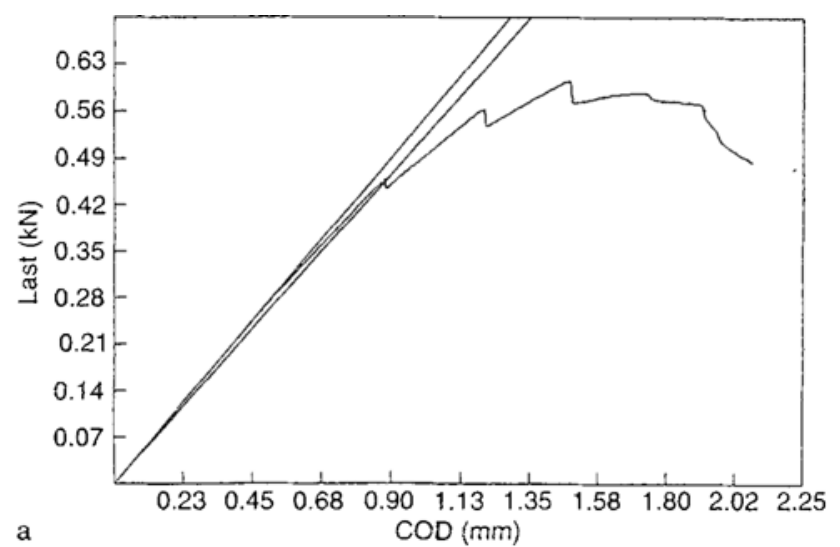
$\mathrm{COD}(\mathrm{mm})$

b
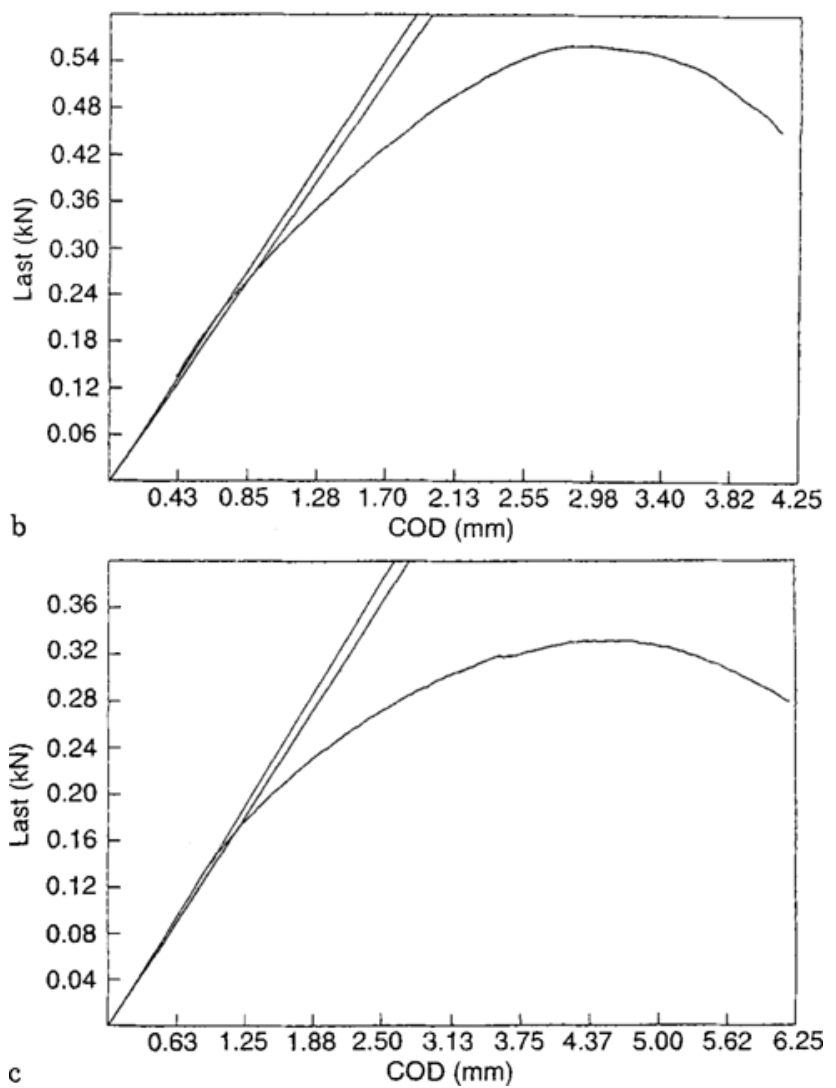

Bild 3a-c. Typische Rißaufweitungsdiagramme yon MDF bei Variation der Feuchtigkeit (Rohdichte: $489 \mathrm{~kg} / \mathrm{m}^{3}$ ); a) $50 \%$ rel. Luftfeuchtigkeit; b) $85 \%$ rel. Luftfeuchtigkeit c) $95 \%$ rel. Luftfeuchtigkeit

Fig. 3a-c. Typical plots of load vs. crack displacement of MDF with different moisture contents; a) 50\% rel. humidity; b) $85 \%$ rel. humidity; c) $95 \%$ rel. humidity stellt werden. Die Streuung der Einzelwerte ist infolge der ausgeprägten Individualität der Einzelproben zu hoch.

Beim Bruchvorgang selbst kommt es offensichtlich zum Herausziehen der Fasern aus dem Faserverbund (Bild 5 und 6). Die Abbildung 5 zeigt die Aufnahme eines solchen Risses, Abbildung 6 die Bruchfläche. Ein typischer, interpartikulärer Holzbruch, wie er bei Spanplatten zu beobachten ist, ist bei den untersuchten MDF-Platten nicht nachweisbar. Die Vielzahl der auftretenden Schallsignale ist offensichtlich auf das Herausziehen der Fasern aus dem Faserverbund, zwischenpartikuläre Reibungen sowie Fa-
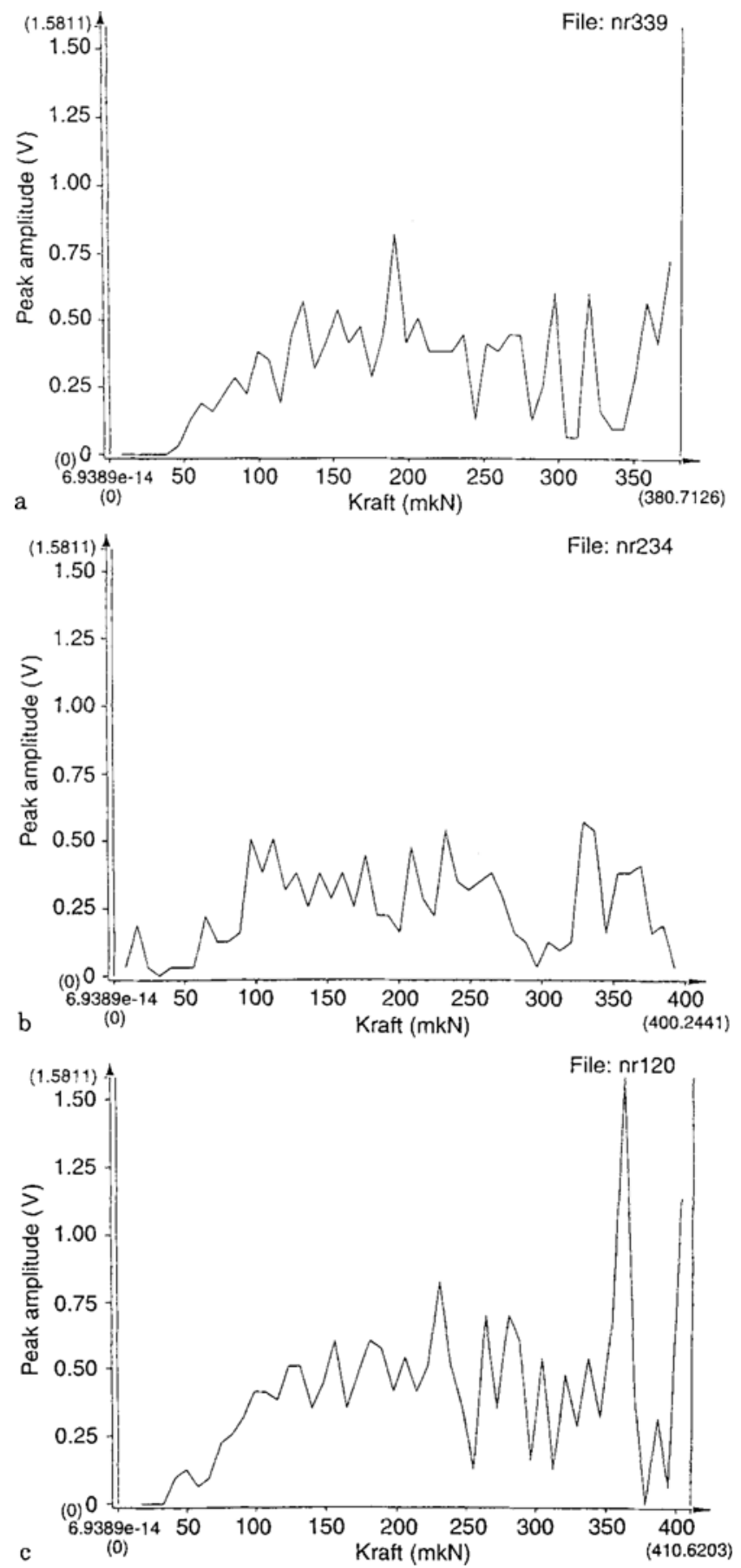

Bild 4a-c. Schallemissionsignale während der Belastung (PeakAmplitude über der Kraft); a) Rohdichte $489 \mathrm{~kg} / \mathrm{m}^{3}$; b) Rohdichte $\left.596 \mathrm{~kg} / \mathrm{m}^{3} ; \mathrm{c}\right)$ Rohdichte $584 \mathrm{~kg} / \mathrm{m}^{3}$

Fig. 4a-c. Acoustic emission signals during the test (Peak amplitude vs strength) a) density $489 \mathrm{~kg} / \mathrm{m}^{3}$; b) density $596 \mathrm{~kg} / \mathrm{m}^{3}$; c) density $684 \mathrm{~kg} / \mathrm{m}^{3}$ 


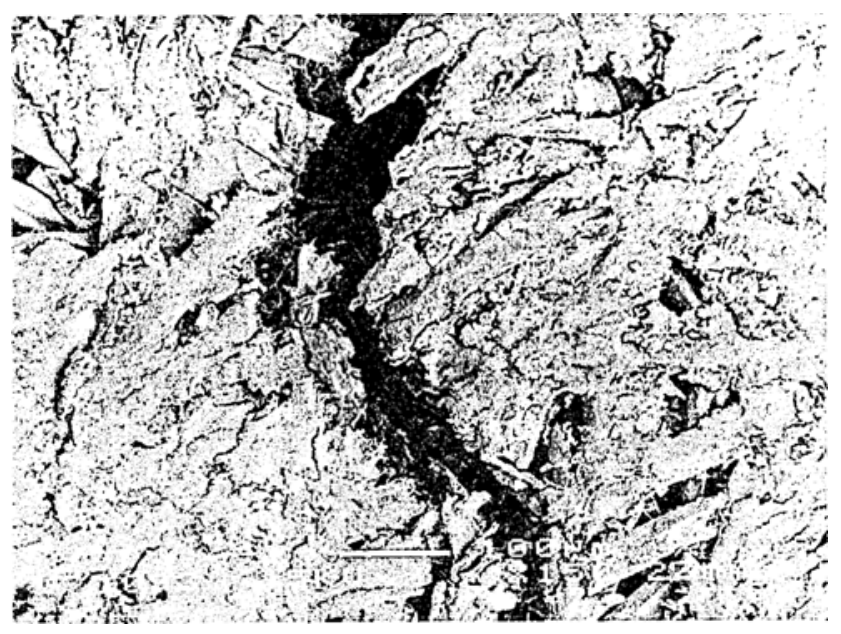

Bild 5. REM-Aufnahme eines Risses von MDF (Foto: T. Zimmermann, EMPA Dübendorf)

Fig. 5. Fracture of MDF examined by SEM (photo made by $T$. Zimmermann, EMPA Dübendorf)

serbrüche zurückzuführen. Eine exakte Zuordnung der Schallsignale zu deren Quellen ist aber nicht möglich.

\section{4}

\section{Literatur}

ASTM E 399 (1994) Standard test method for plain-strain fracture toughness of metallic materals, Annual Book of ASTM Standards, American Society for Testing Materials, Philadelphia

Boström L (1992) Method for determination of softening behavior of wood and the applicability of a nonlinear fracture mechanics model. Dissertation, Lund/Schweden

Erhart RJA, Stanzl-Tschegg SE, Tschegg EK (1996) Fracture properties of particleboard-Influence of specimen geometry. Proceedings of the 1996 International Conference on Wood Mechanics. FMPA-Otto-Graf-Institut, Stuttgart

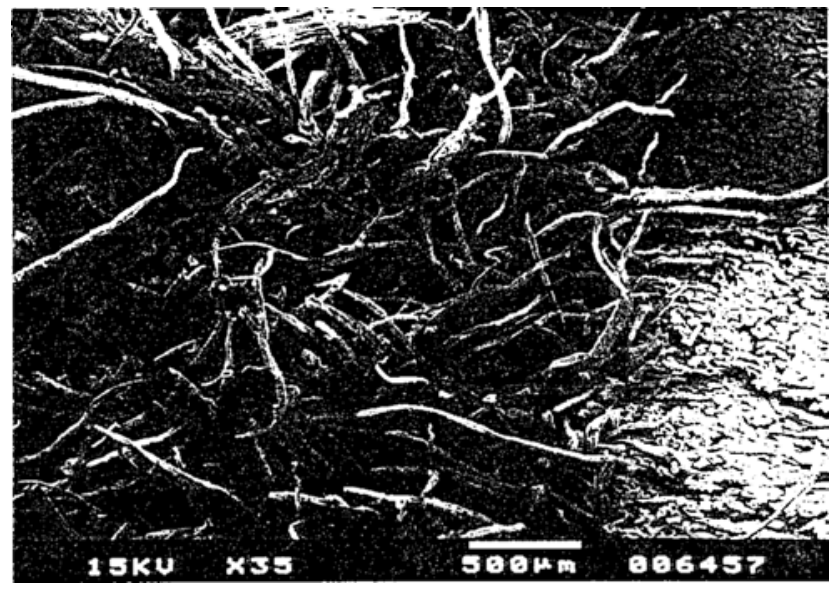

Bild 6. REM- Aufnahme einer Bruchfläche von MDF (Foto: E. Bäucker, TU Dresden)

Fig. 6. Fracture surface of MDF examined by SEM (photo made by E. Bäucker, TU Dresden)

Logemann M (1991) Abschätzung der Tragfähigkeit von Bauteilen mit Ausklinkungen und Durchbrüchen. VDI-Verlag, Reihe 4 Bauingenieurwesen, Nr. 102. Düsseldorf

Niemz P, Schädlich S (1992) Untersuchungen zum Einfluß der Struktur auf die Bruchzähigkeit von Spanplatten. Holz RohWerkstoff 50:389-391

Stanzl-Tschegg SE, Tan DM, Tschegg EK (1995) New splitting method for wood fracture characterization. Wood Sci. Technol. 29:30-50

Valentin GH, Boström L, Gustafsson PJ, Ranta-Maunus A (1991) Application of fracture mechanics to timber structures RILEM state-of-the art report. Valtio Teknillinen Tutkimuskeskus Statens Tekniska Forskingscentral Technical Research Centre of Finland, Espoo 